\title{
Effect of a period of Selected SMR/Theta Neurofeedlback Training on Visual and Auditory Reaction Time in Veterans and Disabled Athletes
}

\section{ART ICLE INFO}

\section{Article Type}

Original Research

\section{Authors}

Parsaee S. ${ }^{1} M A$,

Alboghbish S.* MA,

Abdolahi H. ${ }^{2} M A$,

Alirajabi R. ${ }^{3} M D$,

Anbari A. ${ }^{4} P h D$

How to cite this article
Parsaee S, Alboghbish S, Abdola-
hi H, Alirajabi R, Anbari A. Effect
of a period of Selected SMR/The-
ta Neurofeedback Training on Vi-
sual and Auditory Reaction Time
in Veterans and Disabled Athletes.
Iranian Journal of War \& Public
Health.2018;10(1):15-20.

\section{A B S T R A C T}

Aims Reaction time is one of the most accurate indicators for evaluating speed and efficiency of decision making in different situations, especially sport situations. This study aimed to investigate the effect of neurofeedback training on the visual and auditory reaction time of veterans and disabled athletes.

Materials \& Methods In this semi-experimental study with pre-test-post-test design in 2017, 16 veterans and disabled people in Shiraz, were selected by convenience sampling. They were randomly divided into two experimental and control groups. The experimental group participated in 15 sessions of neurofeedback training with Vilistus tool and enhancement sensorimotor rhythm (SMR) protocol and declining Theta. All the participants performed a simple and selective reaction time in visual and auditory, before and after the beginning of the training. Data were analyzed by SPSS 21 using mixed analysis of variance, analysis of variance with repeated measure and LSD post hoc tests.

Findings Neurofeedback training reduced reaction time in visual simple $(\mathrm{p}=0.007)$, auditory simple $(\mathrm{p}=0.001)$, visual selective $(\mathrm{p}=0.003)$, and auditory selectivity $(\mathrm{p}=0.0001)$.

Conclusion Neurofeedback training is effective on improving brain functions for simple and selective reaction time during visual and auditory in veterans and disabled athletes.

Keywords Brain Waves; Neurofeedback; Reaction Time; Veterans; Disabled

\section{I T A T I O N L I N K S}

*Sport Psychology Department, Physical Education Faculty, Shahid Chamran University of Ahvaz, Ahvaz, Iran

${ }^{1}$ Sport Psychology Department, Physical Education Faculty, Shahid Chamran University of Ahvaz, Ahvaz, Iran

${ }^{2}$ Political Science Department, Humanities Faculty, Ahvaz Branch, Islamic Azad University, Ahvaz, Iran ${ }^{3}$ Police Management Department, Basic Sciences Faculty, Amin of Police Sciences University, Tehran, Iran ${ }^{4}$ Sociology Department, Humanities Faculty, Dehaghan Branch, Islamic Azad University, Isfahan, Iran

\section{Correspondence}

Address: Department Sports Psychology, Physical Education \& Sport Sciences Faculty, Shahid Chamran University of Ahvaz, Golestan Street, Ahvaz, Iran

Phone: +98 (061) 3332341

Fax: +98 (061) 3332341

albogbish@gmail.com

\section{Article History}

Received: August 03, 2017

Accepted: October 17, 2017

ePublished: January 11, 2018

\begin{abstract}
[1] Effects of the amount and intensity of exercise on plasma ... [2] Participant barriers of Kerman provinces wheel chaired people in recreational ... [3] Effect of neuro-linguistic programming on mental toughness in veteran and ... [4] The effect of life skills training on the neoplasm of women with physical-mental ... [5] Nicotine deprivation effects on the dissociated components of ... [6] Effect of foreperiod duration and handedness on simple and choice auditory reaction time among ... [7] EEG-neurofeedback for optimizing performance. I: A review of cognitive and affective outcome in ... [8] A comparison of the effects of neurofeedback and physical practices on performance and retention ... [9] QEEGguided neurofeedback in the treatment of obsessive ... [10] Effect of neurofeedback on motor recovery of a patient with brain injury: A case study and its implications for ... [11] The effect of selected Hatha Yoga and pranayama exercise on motor and cognitive function in ... [12] Effect of neurofeedback training on improvement of reaction time in elderly ... [13] The effect of neurofeedback (SMR training) on performance and ... [14] Effects of SMR and theta/beta neurofeedback on reaction times, spatial ... [15] Increasing individual upper alpha power by neurofeedback improves ... [16] Shutting down sensorimotor interferences after stroke: A proof-of-principle ... [17] Shutting down sensorimotor interference unblocks the networks for stimulus processing ... [18] Assiament facilitate and significant interference of stroop effect on ... [19] EEG biofeedback of low beta band components: Frequency-specific effects on variables ... [20] Effectiveness of neurofeedback training in cognitive ... [21] The effect of gamma enhancing neurofeedback on the control of feature bindings and intelligence ... [22] The effect of individual upper alpha band enhancing neurofeedback on reaction-time as an indicator of short-term memory in ... [23] Controlled evaluation of a neurofeedback training of slow cortical potentials in children with attention deficit/hyperactivity ... [24] Comparison between auditory and visual simple ... [25] Peroneal reaction time after ankle sprain: A systematic ... [26] Effect of pranayama on visual and auditory ... [27] The effect of neurofeedback training on attention rate in ... [28] Handbook of neurofeedback: Dynamics and clinical .. [29] Creativity comparison between students who studied life skills courses and those...
\end{abstract}




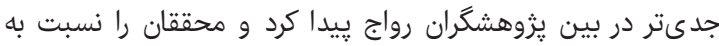

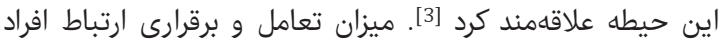

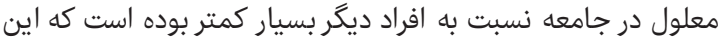

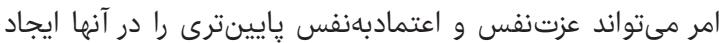

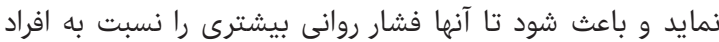

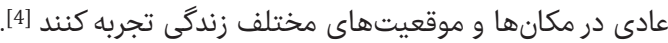

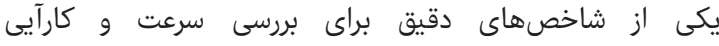

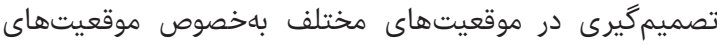

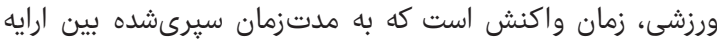

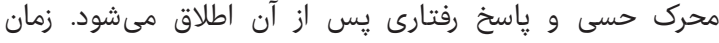

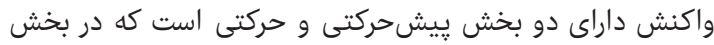

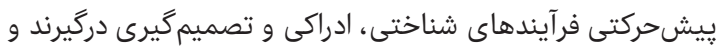

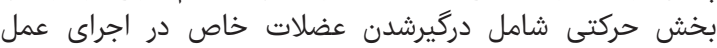

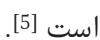

در بسيارى از مهارتهاى ورزشى يا تمرينات درمانى در افراد

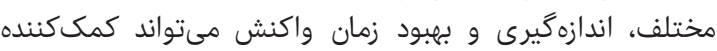

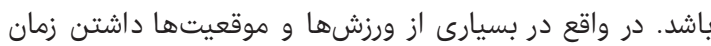

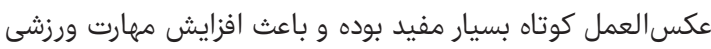

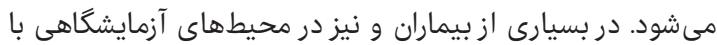

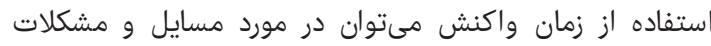

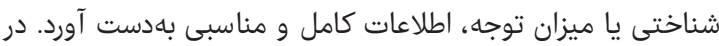

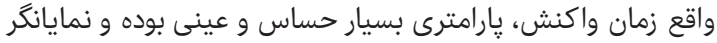

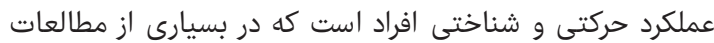

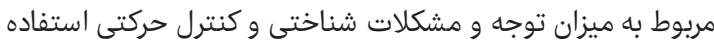

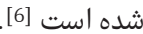

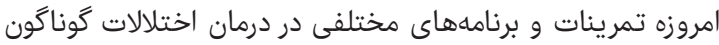

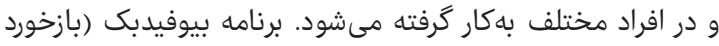

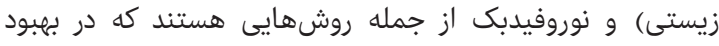

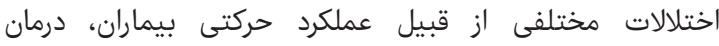

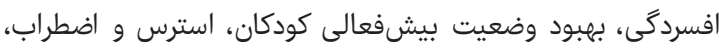

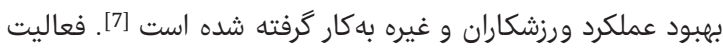

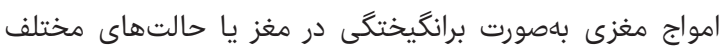

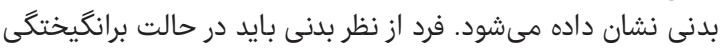

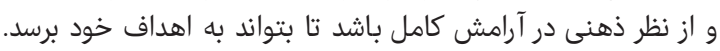

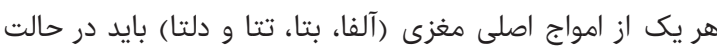

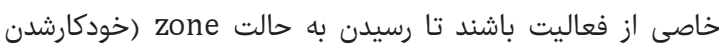

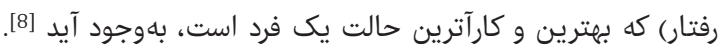

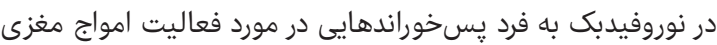

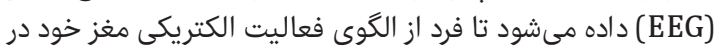

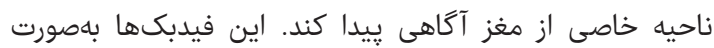

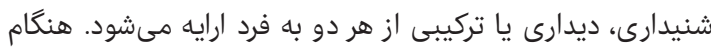

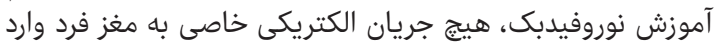

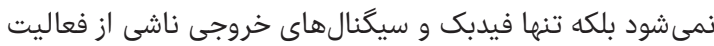

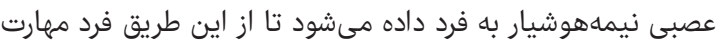

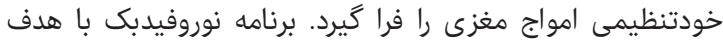

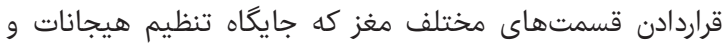

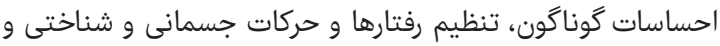

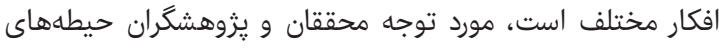

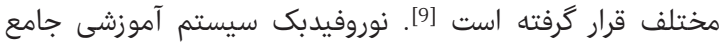

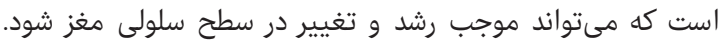

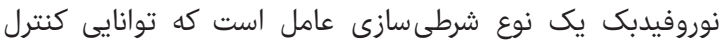

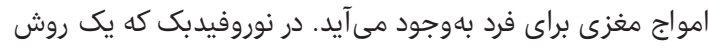

\section{تاثير يك دوره تمرينات منتخب نوروروفيدبك

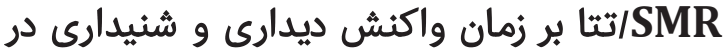

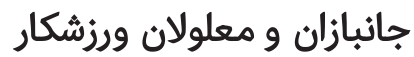

MA سجاد بِارسايى

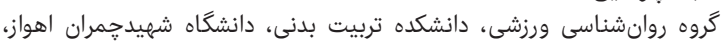
اهواز، ايران

MA " سعيد البوغبيش ايران

كَروه روانشناسى ورزشى، دانشكده تربيت بدنى، دانشگًاه شهيدجمران اهواز،

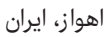

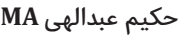
كروه علوم سياسى، دانشكده علوم انسانى، واحد اهواز، دانشكاه آزاد اسلامى،

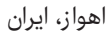

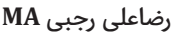

كَروه مديريت انتظامى، دانشكده علوم پِايه، دانشًاه علوم انتظامى امين، تهران،

على عنبرى PhD

كَروه جامعهشناسى، دانشكده علوم انسانى، واحد دهاقان، دانشكاه آزاد اسلامى،

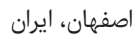

קكيده اهُهداف: يكى از شاخصهاى دقيق براى بررسى سرعت و كارآيى تصميمگيرى در

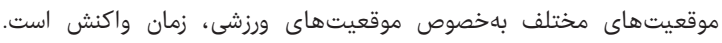

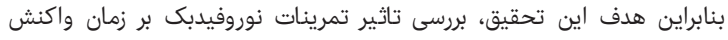

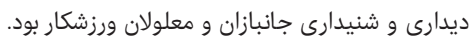

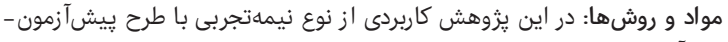

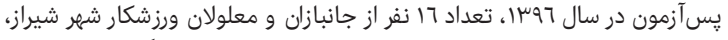

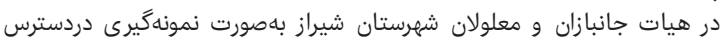

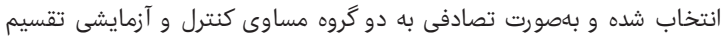

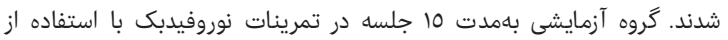

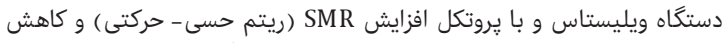

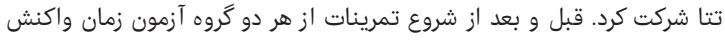

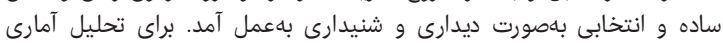

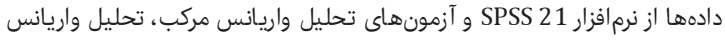

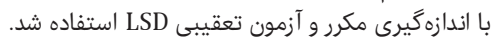

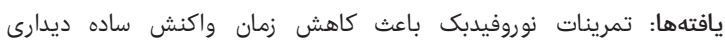

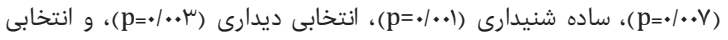

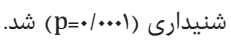

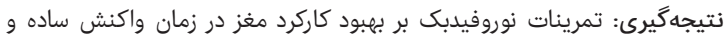

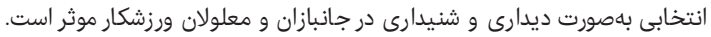
كليدوازمها: امواج مغزى، نوروفيدبك، زمان وان داكنش، جانبازان، معلوانان

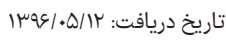

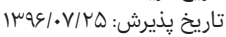

"نويسنده مسئول:

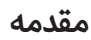

برآورد كامل تعداد افراد دجار معلوليتهاى جسمى مخار - حركتى بسيار

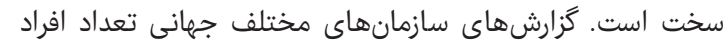

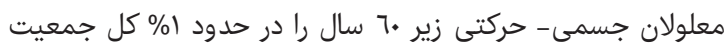

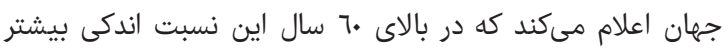

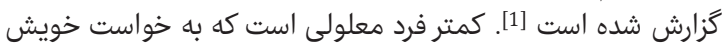

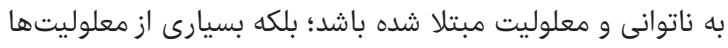

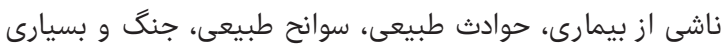

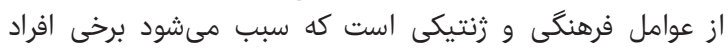

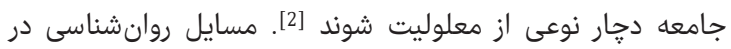

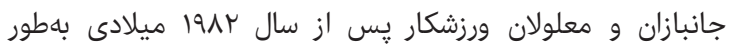




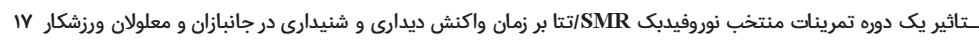

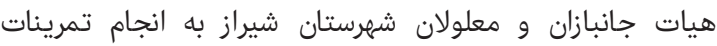

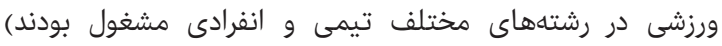

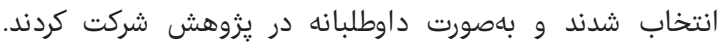

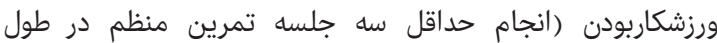
هفته)، نداشتن اختلالات روانى و عدم انجام اندام جراحى در ناحيه سر سر

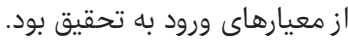

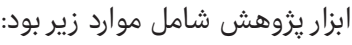

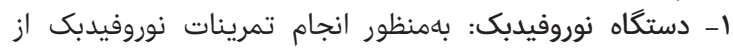

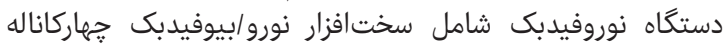

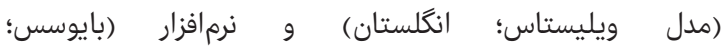

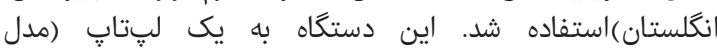

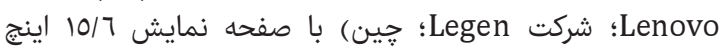

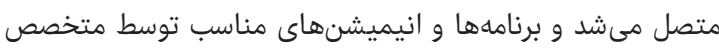

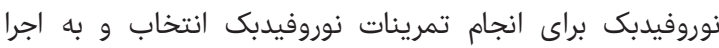

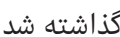

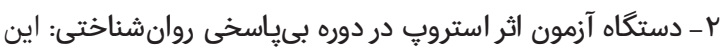

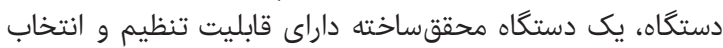

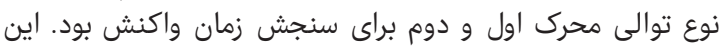

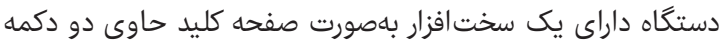

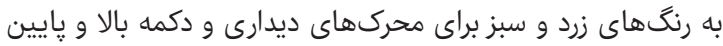

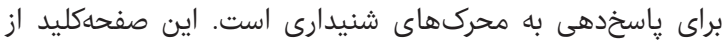

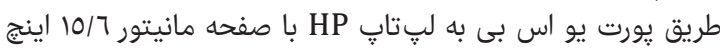

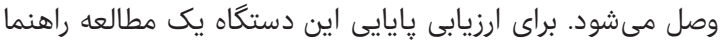

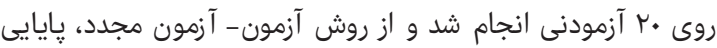

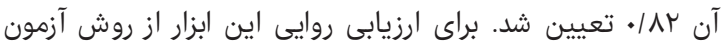

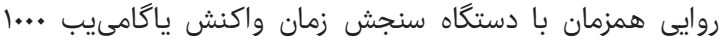

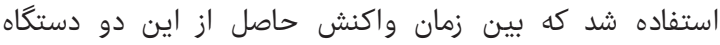

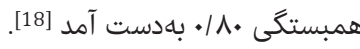

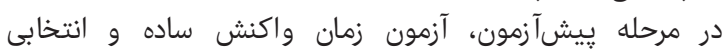

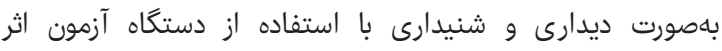

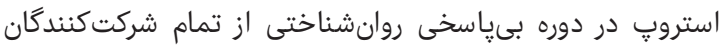

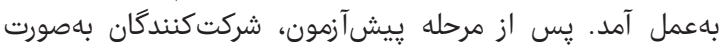

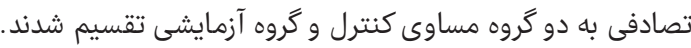

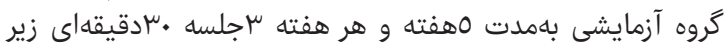

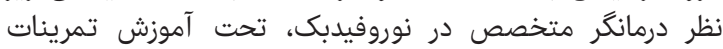

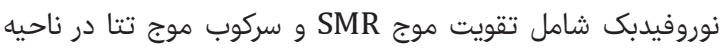

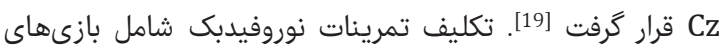

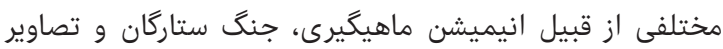

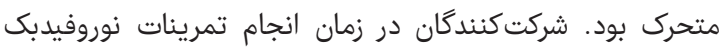

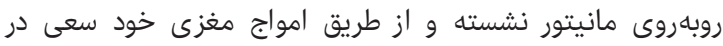

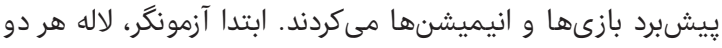

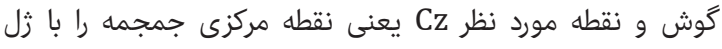

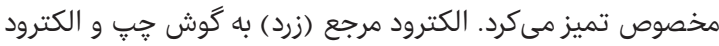

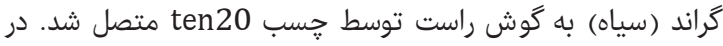

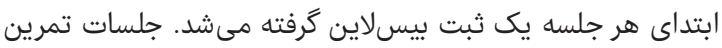

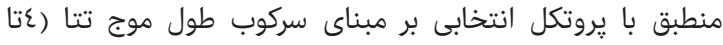

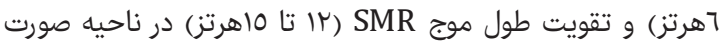

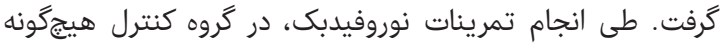

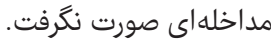

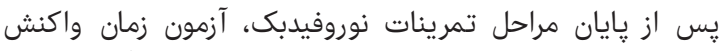

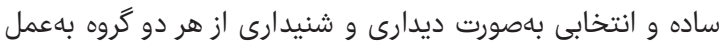

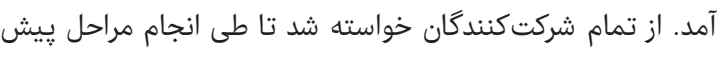

ايمن و غيرتهاجمى است، سنسورهايى كه الكترود ناميده مى شوند

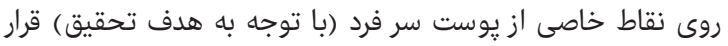

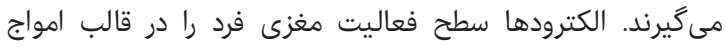

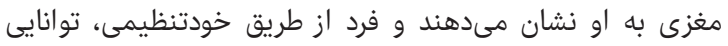
هدايت اين امواج را ييدا مىكند مئد [10].

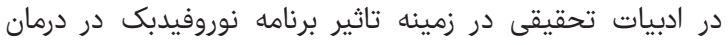

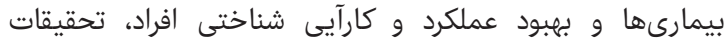

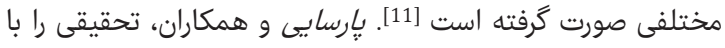

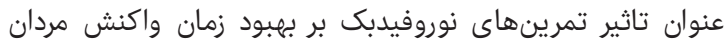

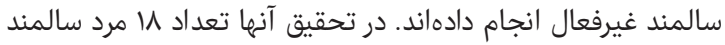

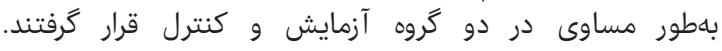

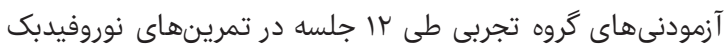

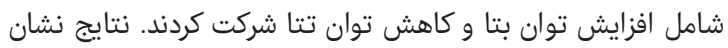

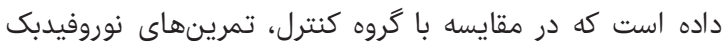

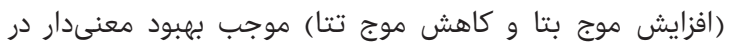

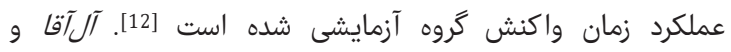

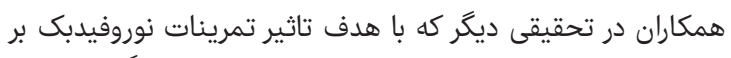

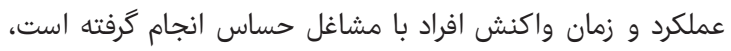

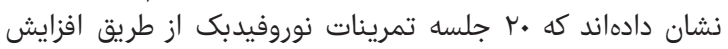

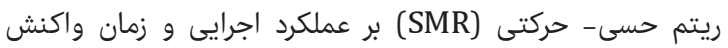

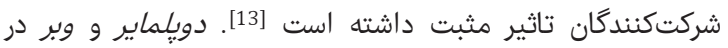

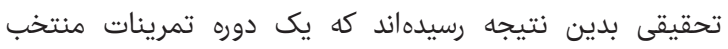

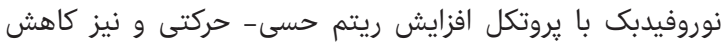

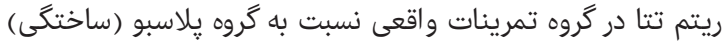

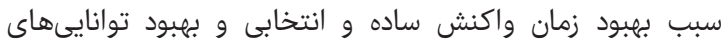

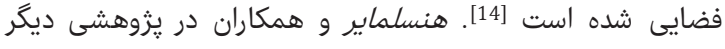

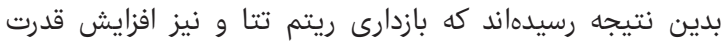

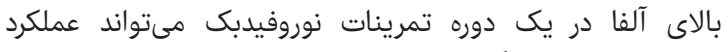

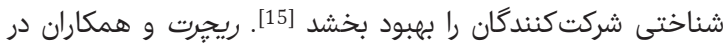

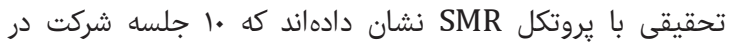

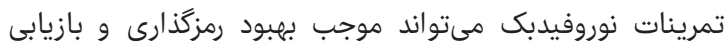

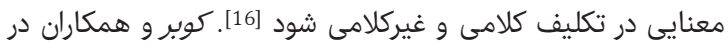

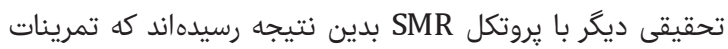

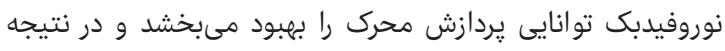

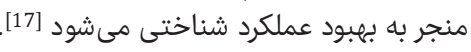

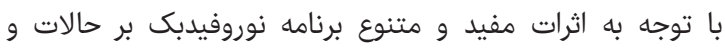

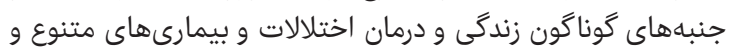

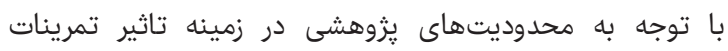

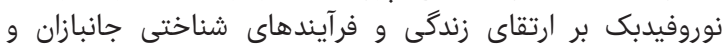

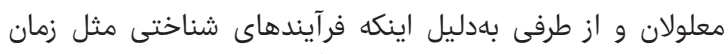

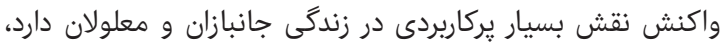

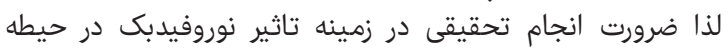

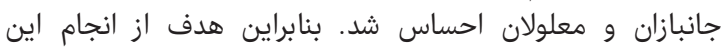

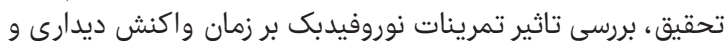
شنيدارى جانبازان و معلولان ورزشكار بود. توريد

\section{مواد و روشها}

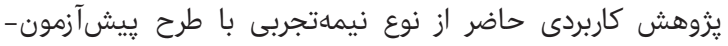

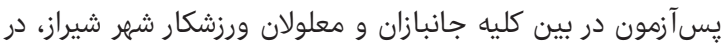

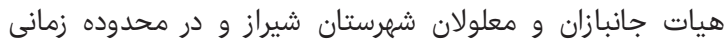

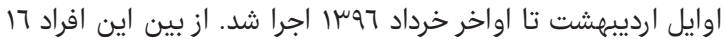

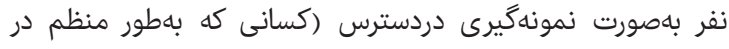


شناختى تنيسورهاى مبتدى بدين نتيجه دست يافتهاند كه

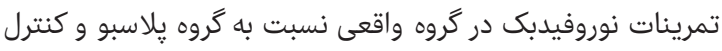

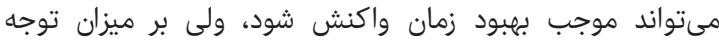

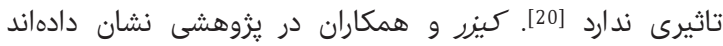

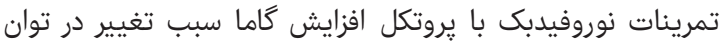

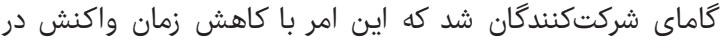

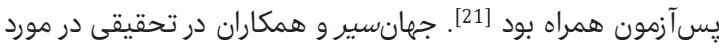

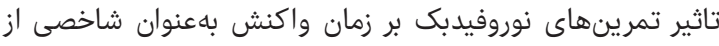

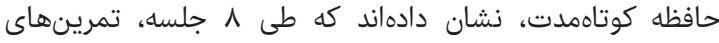

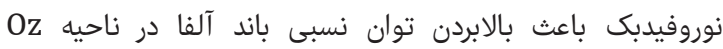

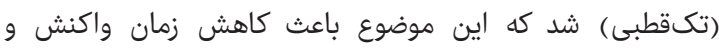

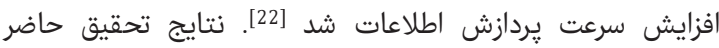

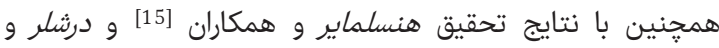

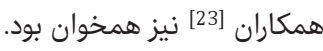

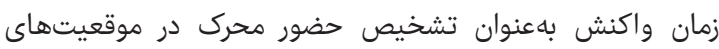

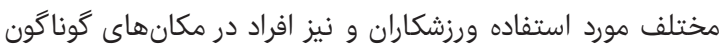

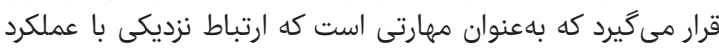

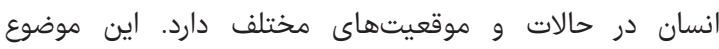

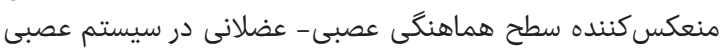

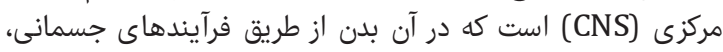

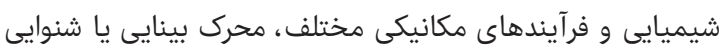

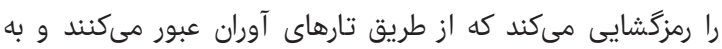

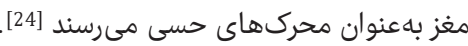

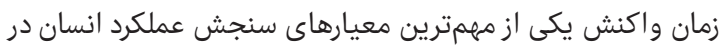

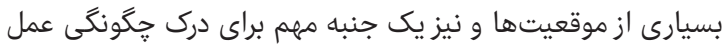

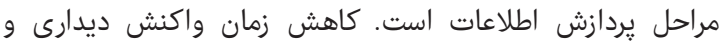

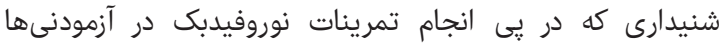

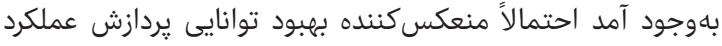

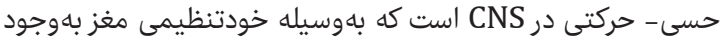

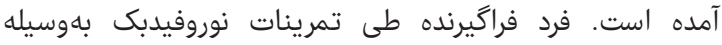

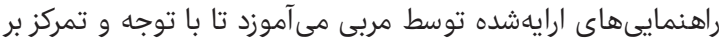

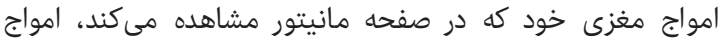

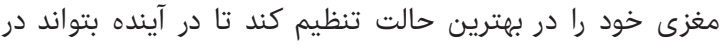

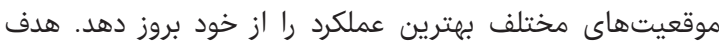

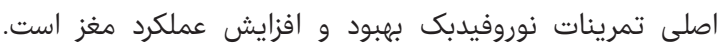

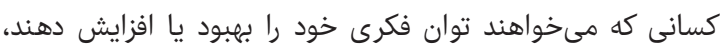
نوروفيدبك يكى از راههاى كمك كننده و بى خطر در در اين زمينه است

[7, 25, 26]

از طرفى، شركت در تمرينات نوروفيدبك، سبب اصلاح عملكرد بد بد مريد

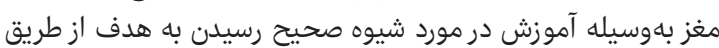

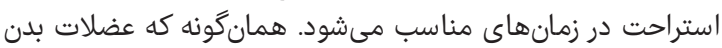

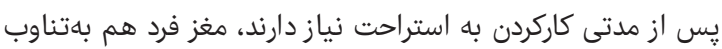

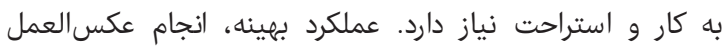

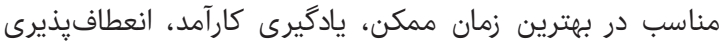

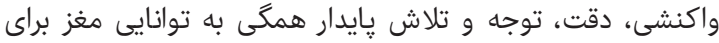

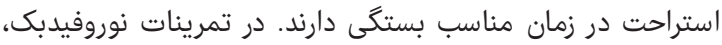

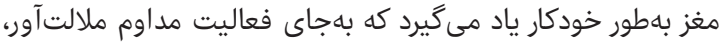

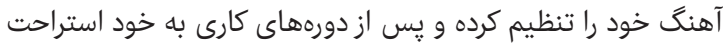

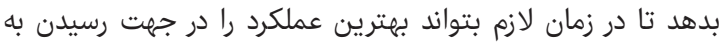

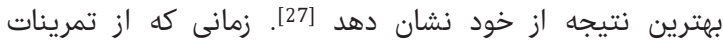
نوروفيدبك بهمنظور بجبود فرآيندهاى شناختى (از قبيل زمان ان تبنان

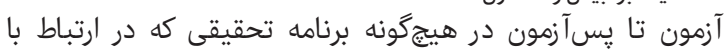

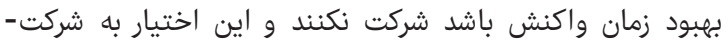

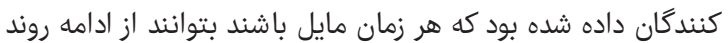

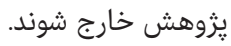

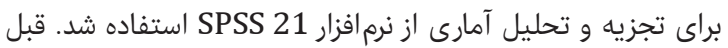

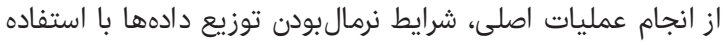

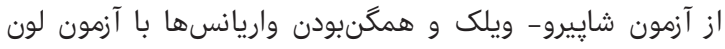

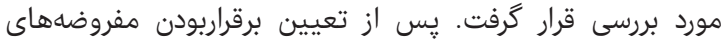

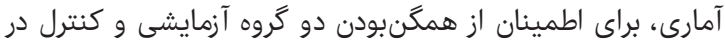

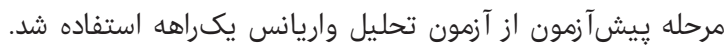

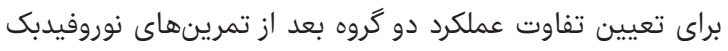

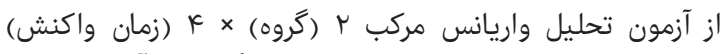

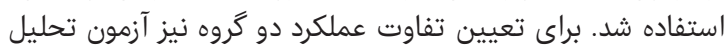
واريانس با اندازهكيرى مكرر و آزمون تعقيبى LSD مورد استفاده

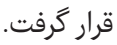

يافتهها

بين دو كروه آزمايشى و كنترل در مرحله يِيشآزمون در هر جهار

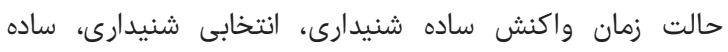

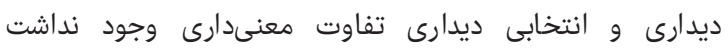
(p>. $1 \cdot 0)$

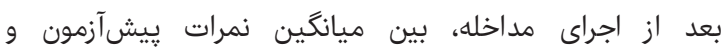

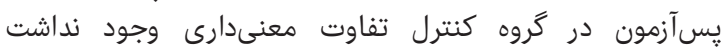

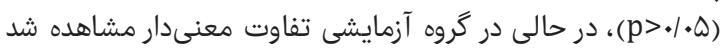

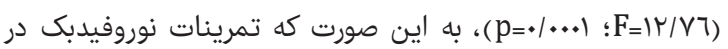

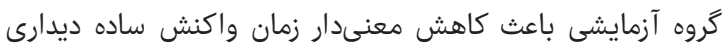

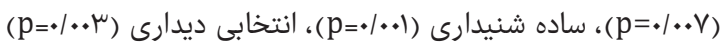

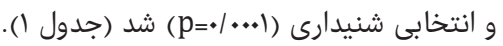

جدول () مقايسه ميانكَين آمارى زمان واكنش ساده و انتخابى (بر حسب هزارم

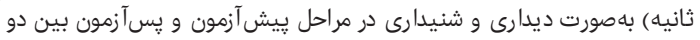
گروه آزمايشى و كنترل (هر گَروه 1 نفر )

\begin{tabular}{|c|c|c|}
\hline يس آزمون & ي ييش آزمون & متغيرها \\
\hline & & زمان واكنش شنيدارى ساده \\
\hline Hrr/qY $\pm 9 \cdot / I V$ & $\mu_{T V / / T \pm \Lambda T / \wedge V}$ & گروه آزمايشى \\
\hline 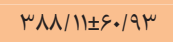 & $\mu \varepsilon \mu / / \gamma \pm 7 . / \varepsilon\rceil$ & كروه كنترل \\
\hline & & زمان واكنش شنيدارى انتخابى \\
\hline$r \wedge N / 1 T \pm \kappa_{*} / \mu \Delta$ & $711 / 17 \pm . / 9 \varepsilon$ & كروه آزمايشى \\
\hline$G K G / T \cdot \pm V \mu / \Delta q$ & $T / N / r r \pm T T / V O$ & كروه كنترل \\
\hline & & زمان واكنش ديدارى ساده \\
\hline$\mu \cdot \mu / 1 \Delta \pm \mu \mu / \mu q$ & 每 & گروه آزمايشى \\
\hline 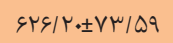 & $T A V / T H \pm 7 T / O V$ & كروه كنترل \\
\hline & & زمان واكنش ديدارى انتخابى \\
\hline$s \mid Q / / K \pm K \varepsilon / . F$ & $V T N / T I \pm V H / A r$ & كروه آزمايشى \\
\hline$\Delta I r / r Y \pm V H / V V$ & $\Lambda \mu O / \varepsilon \cdot \pm T \cdot / \mu$. & كروه كنترل \\
\hline
\end{tabular}

بحث

هدف از انجام اين يزوهش برورى ترو تاثير يك دوره تمرينات منتخب

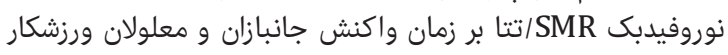

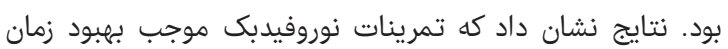

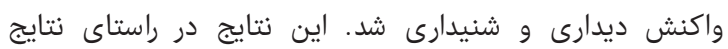

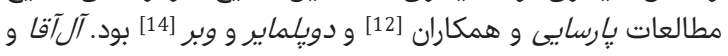
همكاران در تحقيقى با عنوان تاثير تمرينات نوروفيدبك بر بران عملكرد 


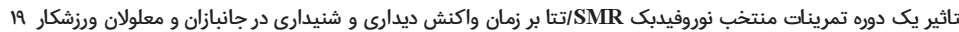

2017;9(2):61-6. [Persian]

4- Moradi A, Kalantari M. The effect of life skills training

on the neoplasm of women with physical-mental

disability. Res Except Child. 2006;19(1):559-76.

[Persian]

5- Marzilli TS, Hutcherson AB. Nicotine deprivation

effects on the dissociated components of simple reaction time. Percept Mot Skills. 2002;94(3):985-95.

6- Rezaeimanesh S, Norouzi E, Parsaei S, Shetab Boushehri N, Norouzi Seyed Hossieni R, Gonzalez Vega N. Effect of foreperiod duration and handedness on simple and choice auditory reaction time among the older people. Salmand. 2017;11(4):528-37. [Persian]

7- Gruzelier JH. EEG-neurofeedback for optimizing performance. I: A review of cognitive and affective outcome in healthy participants. Neurosci Biobehav Rev. 2014;44:124-41.

8- Salehi M, Amini H, Mohammadzade H. A comparison of the effects of neurofeedback and physical practices on performance and retention of dart throw skill. Dev Motor Learn. 2016;25:451-67. [Persian]

9- Hammond DC. QEEG-guided neurofeedback in the treatment of obsessive compulsive disorder. I Neurotherapy. 2003;7(2):25-52.

10- Wing K. Effect of neurofeedback on motor recovery of a patient with brain injury: A case study and its implications for stroke rehabilitation. Top Stroke Rehabil. 2001;8(3):45-53.

11- Khazaei AA, Yousefi B, Kahrizi N. The effect of selected Hatha Yoga and pranayama exercise on motor and cognitive function in elderly women. Int J Sport Stud. 2014;4(5):547-53.

12- Parsaei S, Shetab Bushehri N, Alboghebish S, Rezaeimanesh S, Barati P. Effect of neurofeedback training on improvement of reaction time in elderly, passive males. Salmand. 2017;11(4):550-7. [Persian]

13- Nabavi Aleagha F, Naderi F, Heidarei A, Nazari M, Nicksirat A, Avakh F. The effect of neurofeedback (SMR training) on performance and reaction time of individuals who undertake difficult tasks. Ebnesina. 2014;15(4):36-41. [Persian]

14- Doppelmayr M, Weber E. Effects of SMR and theta/beta neurofeedback on reaction times, spatial abilities, and creativity. J Neurother. 2011;15(2):115-29. 15- Hanslmayr S, Sauseng P, Doppelmayr M, Schabus M, Klimesch W. Increasing individual upper alpha power by neurofeedback improves cognitive performance in human subjects. Appl Psychophysiol Biofeedback. 2005;30(1):1-10.

16- Reichert JL, Kober SE, Schweiger D, Grieshofer P, Neuper C, Wood G. Shutting down sensorimotor interferences after stroke: A proof-of-principle SMR neurofeedback study. Front Hum Neurosci. 2016;10:348. 17- Kober SE, Witte M, Stangl M, Väljamäe A, Neuper C, Wood G. Shutting down sensorimotor interference unblocks the networks for stimulus processing: An SMR neurofeedback training study. Clin Neurophysiol 2015;126(1):82-95.

18- Alboghebish S, Shetabbushehri N, Daneshfar A, Abedanzade R. Assiament facilitate and significant interference of stroop effect on psychological refractory period. J Neuropsychol. 2017;2(7):91-104. [Persian] 19- Egner T, Gruzelier JH. EEG biofeedback of low beta band components: Frequency-specific effects on variables of attention and event-related brain potentials. Clin Neurophysiol. 2004;115(1):131-9.

20- Nabavi Al Agha F, Naderi F, Heidarei A, Ahadi H,
واكنش) استفاده شود و اين دو با هم تركيب شوند، مىتوان انتظار

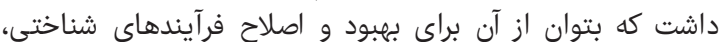
ذهنى و حتى جسمانى در افراد مختلف استفاده كرد [28].

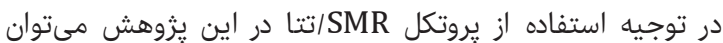

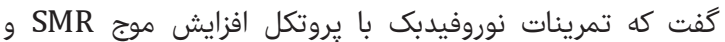
كاهش موج تتا سبب استحكامبخشيدن به ذهن، مغز و در نتيجها

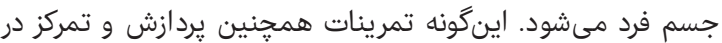

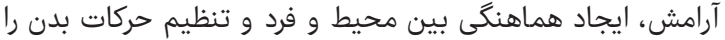

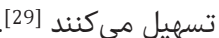

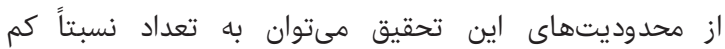
آزمودنىها اشاره كرد كه بهدليل محدوديتهاى موجود در اين زمينه و عدم دسترسى به تعداد آزمودنى مورد نياز در اين زمينه صورد

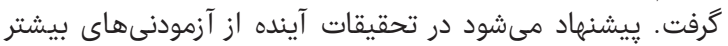

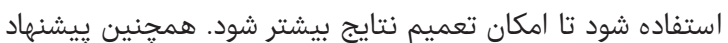

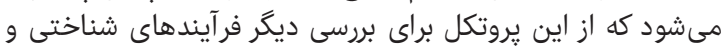

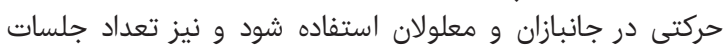

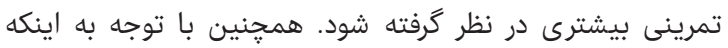

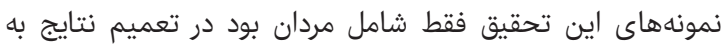

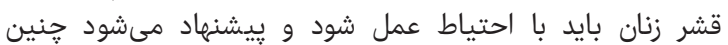
تحقيقى روى آزمودنىهاى زن نيز انجام شود.

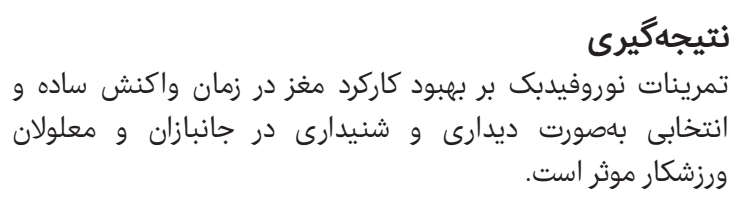

تشكر و قدردانى: در يايان، نويسندگان اين مقاله بر خود لازم

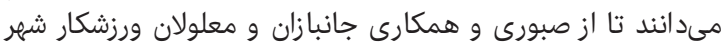
شيراز كه در اين تحقيق شركت كردند، تشكر و قدردانى بهاعمل آورند. تاييديه اخلاقى: تمام شركتكنندكان با اعلام رضايت كامل شفاهى

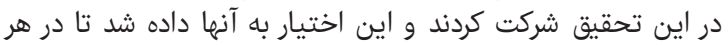

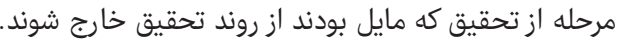

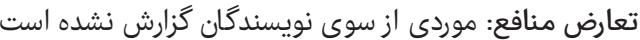

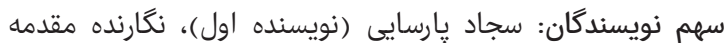

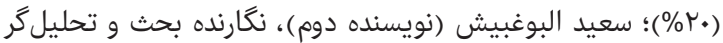

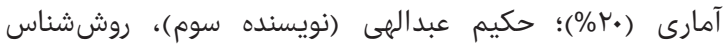

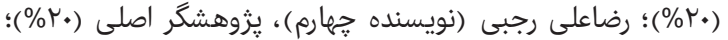

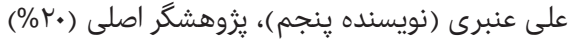

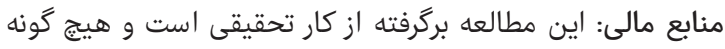
حمايت مالى از سوى نهاد يا سازمانى انجام نگرفته است.

1- Kraus WE, Houmard JA, Duscha BD, Knetzger KJ, Wharton MB, McCartney JS, et al. Effects of the amount and intensity of exercise on plasma lipoproteins. N Engl J Med. 2002;347(19):1483-92.

2- Pourranjbar M, Keshavarz L, Sharifian E, Farahani A. Participant barriers of Kerman provinces wheel chaired people in recreational activities.J Health Dev. 2014;3(2):175-80. [Persian]

3- Akbarzade B, Ahar S, Dadashzade M. Effect of neurolinguistic programming on mental toughness in veteran and disabled athletes. Iran J War Public Health. 
2010;1(1):30-2.

25- Hoch MC, Mckeon PO. Peroneal reaction time after ankle sprain: A systematic review and meta-analysis. Med Sci Sports Exerc. 2014;46(3):546-56.

26- Borker AS, Pednekar JR. Effect of pranayama on visual and auditory reaction time. Indian J Physiol Pharmacol. 2003;47(2):229-30.

27- Hashemian P, Farrokhi A, Mirifar A, Keihani M, Sajadi AR. The effect of neurofeedback training on attention rate in proficient athletics. J Funda Ment Health. 2014;15(60):312-8. [Persian]

28- EvansJR, Othmer SF, Maldonado Rubi CM, Joffe D, Othmer S, JR TT, et al. Handbook of neurofeedback: Dynamics and clinical application. Boca Raton: CRC Press; 2007. pp. 321-37.

29- Bashardoost Tajalli F, Zandi Z. Creativity comparison between students who studied life skills courses and those who didn't. Procedia Soc Behav Sci. 2010;5:1390-5.
Nazari MA. Effectiveness of neurofeedback training in cognitive performance. J Clin Sci Andishe Raftar.

2013;7(26):27-36. [Persian]

21- Keizer AW, Verschoor M, Verment RS, Hommel B. The effect of gamma enhancing neurofeedback on the control of feature bindings and intelligence measures. Int J Psychophysiol. 2010;75(1):25-32.

22- Jahanseir M, Firooz Abadi SM, Ghoshouni M, Motie Nasrabadi A. The effect of individual upper alpha band enhancing neurofeedback on reaction-time as an indicator of short-term memory in women employees. Iran South Med J. 2014;17(5):834-46. [Persian]

23- Drechsler R, Straub M, Doehnert M, Heinrich H, Steinhausen HC, Brandeis D. Controlled evaluation of a neurofeedback training of slow cortical potentials in children with attention deficit/hyperactivity disorder (ADHD). Behav Brain Funct. 2007;3:35.

24- Shelton J, Kumar GP. Comparison between auditory and visual simple reaction times. Neurosci Med. 